\title{
A Survey Paper on Particle Swarm Optimization based Routing Protocols in Mobile Ad-Hoc Networks
}

\author{
Shruti Dixit \\ UIT RGPV, Bhopal
}

\author{
Rakesh Singhai, PhD \\ UIT RGPV, Bhopal
}

\begin{abstract}
Swarm Intelligence is an artificial intelligence discipline which is concerned with the design of intelligent multi-agent systems by taking inspiration from the collective behaviours of social insects and other animal societies. They are characterized by a decentralized way of working that mimics the behaviour of the swarm. Swarm Intelligence is a successful paradigm for the algorithm with complex problems. The purpose of the research is to apply swarm intelligence on routing algorithms used in MANET. Swarm intelligence based routing algorithm will more promising for specific nature of ad- hoc networks and will outperform in real scenarios/constraints/environmental conditions and will tune and simulate to get an efficient and effective routing protocol for MANET.
\end{abstract}

\section{Keywords}

MANET, honeybee, Particle swarm optimization, Swarm Intelligence, Ant Colony Optimization (ACO).

\section{INTRODUCTION}

Ad-hoc networks [1] communicate over wireless media between stations directly in a peer to peer fashion without the help of wired base station or access points. Mobile Ad Hoc Networks (MANETs) is one of ad-hoc network communication system which is extensively used. MANETs are built up of a collection of mobile nodes which have no fixed infrastructure. The nodes communicate independently through wireless network without any central control. Routing is the task of directing data packets from a source node to a given destination. The routing is complex procedure due to the dynamic topology, limited process, storing capability, bandwidth constraints and lack of the central control.

Routing protocols for MANETs suffer from certain inherent shortcomings. First the proactive routing schemes used in MANET like Destination Sequenced Distance Vector (DSDV) continuously updates the routing tables of mobile nodes which large portion of the scarce network capacity for exchanging huge chunk of routing table data. This reduces the available capacity of the network for actual data communication. Secondly the on-demand routing protocols like Ad-Hoc On-Demand Distance Vector (AODV) and Dynamic Source (DSR) routing. These protocols launch route discovery, and require the actual communication to be delayed until the route is determined. This is not suitable for real-time data and multimedia communication applications.

Swarm Intelligence (SI) is the collective behaviour of decentralized, self-organized systems, natural or artificial in biological systems. SI is an appealing solution when routing becomes a crucial problem in a complex network scenario, where traditional routing techniques fail completely. SI systems are typically made up of a population of simple agents interacting locally with one another and their environment.

Particle swarm optimization (PSO) [9] is a robust stochastic optimization technique based on the movement and intelligence of swarms. PSO applies the concept of social interaction to problem solving. It uses a number of agents (particles) that constitute a swarm moving around in the search space looking for the best solution. Each particle is treated as a point in an $\mathrm{N}$-dimensional space which adjusts its "flying" according to its own flying experience as well as the flying experience of other particles.

Routing algorithms which are based on swarm intelligence are:-

- Collective system capable of accomplishing difficult tasks in dynamic and varied environments without any external guidance or control and with no central coordination.

- Achieving a collective performance which could not normally be achieved by an individual acting alone.

- Constituting a natural model particularly suited to distribute problem solving.

\section{SWARM INTELLIGENCE}

Swarm Intelligence (SI) is mainly defined as the behaviour of natural or artificial self-organized, decentralized systems. Swarms interact locally with each other or with external agents i.e. environment and can be in the form of bird flocks, ants, bees etc. It is the property of a system whereby the collective behaviour of (unsophisticated) agents interacting locally with their environment cause coherent functional global patterns to emerge. SI provides a basis with which it is possible to explore collective (or distributed) problem solving without centralized control or the provision of a global model.

SI based approaches are nature and bio-inspired. Swarms are abundantly found in nature. In the nature animals form into swarms to search food, build nests, to hunt and avoid being hunted etc. Each individual swarm has simple rule of access to a limited amount of information via its immediate neighbours or local environment. It consists of mainly PSO, Ant colony optimization (ACO) and honeybee paradigms. The population of the potential solution is called as swarm and each individual in the swarm is defined as particle. The particles fly in the swarm to search their best solution based on experience of their own and the other particles of the same swarm. 
The SI based approaches are more promising from other conventional techniques for optimization problems, due to the nature, architecture, topology and functionality of ad hoc networks. SI approaches are more suitable for the routing and energy resources optimization related issues in MANETs.

Bio inspired, SI approaches are more promising for Ad-hoc networks due to the following prominent aspects 1.locality of interactions 2.availability of multiple paths 3 . Self organising 4. Failure backup 5.ability to adapt in a quick and robust way to topological and traffic changes and component failures.

\section{PARTICLE SWARM INTELLIGENCE}

PSO defined a new era in SI. PSO is a population based method for optimization. It is a computational intelligence oriented, stochastic, population-based global optimization technique proposed by Kennedy and Eberhart in 1995.It is inspired by the social behaviour of bird flocking and fish schooling.PSO has been applied to many engineering problems due to its unique searching mechanism, simple concept, computational efficiency and easy implementation. It utilizes a "population" of particles that fly through the problem hyperspace with given velocities.

At each iteration, the velocities of the individual particles are stochastically adjusted according to the historical best position for the particle itself and the neighbourhood best position. Both the particle best and the neighbourhood best are derived according to a user defined fitness function.

The movement of each particle naturally evolves to an optimal or near-optimal solution. PSO is not largely affected by the size and non-linearity of the problem, and can converge to the optimal solution in many problems where most analytical methods fail to converge.

Each particle (population member) in the swarm correspond to a solution in a high-dimensional space with four vectors, its current position, best position found so far, the best position found so far by its neighbourhood and its velocity and adjusts its position in the search space based on the best position reached by itself (pbest) and its neighbour (gbest) during the search process.

Steps in PSO algorithm can be briefed as below:

1. Initialize the swarm by assigning a random position.

2. Estimate the fitness function for each particle.

3. For each individual particle, compare the particle's fitness value with its pbest. If the current value is better than the pbest value, then set this as pbest and the current particle's position, $\mathrm{x}_{\mathrm{i}}$, as $\mathrm{p}_{\mathrm{i}}$.

4. Identify the particle that has the best fitness value. This fitness function identified as gbest,

5. Revise the velocities and positions of all the particles using (1) and (2).

6. Repeat steps $2-5$ until a sufficiently good fitness value is achieved.

Considering a search space of d-dimension and $\mathrm{n}$ particles, whose $i^{\text {th }}$ particle at a particular position $X_{i}\left(x_{i 1}, x_{i 2}, \ldots \ldots x_{i d}\right)$ is moving with a velocity $\mathrm{V}_{\mathrm{i}}\left(\mathrm{v}_{\mathrm{i} 1}, \mathrm{v}_{\mathrm{i} 2}, \ldots \ldots . \mathrm{v}_{\mathrm{id}}\right)$. Each particle is associated with its particular best, $\mathrm{P}_{\mathrm{i}}\left(\mathrm{p}_{\mathrm{i} 1}, \mathrm{p}_{\mathrm{i} 2}, \ldots \ldots . \mathrm{p}_{\mathrm{id}}\right)$ which is defined by its own best performance in the swarm.

Similarly, an overall best performance of the particle with respect to the swarm defined global best is gbest. Each particle tries to modify its position using the following information:

- Current positions,

- Current velocities,

- Distance between the current position and pbest,

- Distance between the current position and gbest.

The movement of the particle is governed by updating its velocity and position attributes.

$\mathrm{V}_{\mathrm{i}}^{\mathrm{t}+1}=\mathrm{wV}_{\mathrm{i}}^{\mathrm{t}} \mathrm{i}+\mathrm{c}_{1} \mathrm{r}_{1}\left(\mathrm{x}_{\text {pbest }}-\mathrm{X}_{\mathrm{i}}^{\mathrm{t}}\right)+\mathrm{c}_{2} \mathrm{r}_{2}\left(\mathrm{x}_{\text {gbest }}-\mathrm{X}_{\mathrm{i}}^{\mathrm{t}}\right)$,

$\mathrm{X}_{\mathrm{i}}^{\mathrm{t}+1}=\mathrm{X}_{\mathrm{i}}^{\mathrm{t}}+\mathrm{V}_{\mathrm{i}}^{\mathrm{t}+1}$

where $\mathrm{w}=$ inertia weight,

$\mathrm{c} 1=$ cognitive acceleration coefficient,

c2 $=$ social acceleration coefficient,

$\mathrm{r} 1$ and $\mathrm{r} 2$ are the random values between 0 and 1 ,

xpbest is the personal best of the particle and xgbest is the global best of the particle. $X_{i}^{t}$ is the current position of $i^{\text {th }}$ particle at iteration t. $\mathrm{V}_{\mathrm{i}}^{\mathrm{t}}$ is the velocity of $\mathrm{i}^{\text {th }}$ particle at iteration t. In standard PSO, a minimization problem is considered which tends to defined a parameter set $\mathrm{x}$ a vector of $\mathrm{m}$ decision variables $: \mathrm{x}=\left(\mathrm{x}_{1}, \mathrm{x}_{2}, \ldots, \mathrm{x}_{\mathrm{m}}\right)^{\mathrm{t}}$ for single objective i.e. Minimize/Maximize $\mathrm{f}(\mathrm{x})$; subject to $\mathrm{x}_{\mathrm{i}}^{(\mathrm{L})} \leq \mathrm{x}_{\mathrm{i}} \leq$ $\mathrm{x}_{\mathrm{i}}^{(\mathrm{U})}, \mathrm{i}=1,2, \ldots ., \mathrm{m}$.

\section{SWARM INTELLIGENCE BASED ROUTING ALGORITHM}

Wireless ad hoc network is a wireless network without any central controlling authority. All nodes should have some fundamental routing potential to make sure packets are delivered to their relevant destinations. While carrying out ad hoc networks, huge complications occur due to the frequent route changes, which is due to the mobility of the nodes and intrusion among nodes. Unequal transport layer and constrained amount of traffic is due to the high packet loss rates and recurrent topological changes in the network. The three well-known problems in ad-hoc networks are the lack of reliable packet delivery due to the intrusion and movement of nodes, incomplete bandwidth due to the channel limitations and constrained node life span caused as a result of small battery size. [1]

Routing protocols are proposed for mobile ad-hoc networks have revealed that the on-demand routing protocols perform better in terms of packet delivery and routing overhead than proactive routing schemes especially in the presence of node mobility. Proactive and hybrid schemes do not perform well in dynamic topologies because of the following two major factors:

1. Slow detection of broken links and 2. Periodic exchange of route updates even when routes are not needed. [2]

The routing protocols under idealistic settings where all nodes function properly. The behaviour of routing protocols where some nodes may be faulty [3]. The paper has proposed that AODV outperforms OLSR in terms of packet delivery ratio while OLSR achieves a much lower end to end delay.

A new cooperative coevolving particle swarm optimization (CCPSO) [4] algorithm addresses the issue of scaling up particle swarm optimization (PSO) algorithms in solving large-scale optimization problems (up to 2000 real-valued 
variables). The proposed CCPSO2 builds on the success of an early CCPSO that employs an effective variable grouping technique random grouping. CCPSO2 adopts a new PSO position update rule that relies on Cauchy and Gaussian distributions to sample new points in the search space, and a scheme to dynamically determine the coevolving subcomponent sizes of the variables. Results and analysis suggest that CCPSO2 is a highly competitive optimization algorithm for solving large-scale and complex multimodal optimization problems.

The designing of optical networks [5] are focused on the wavelength division multiplexing (WDM) technology. This technology divides the huge bandwidth of an optical fiber into different wavelengths, providing different available channels per link of fiber. However, when it is necessary to establish a set of demands, a problem comes up. This problem is known as a routing and wavelength assignment (RWA) problem. Depending on the traffic pattern, two varieties of a RWA problem have been considered in the literature: static and dynamic. Swarm intelligence is very suitable to solve the RWA problem, and presumably that it may obtain such quality results not only in diverse telecommunication optimization problems, but also in other engineering optimization problems.

SI is the local interaction of many simple agents to accede a global goal. SI is based on collective behaviour of social insect colonies for solving different types of problems. A new routing algorithm for MANETs, which combines the idea of ant colony optimization with zone based hierarchical link state (ZHLS) protocol. The algorithm is based on ants jump from one zone to the next zones which contains of the proactive routing within a zone and reactive routing between the zones. The proposed algorithm will improved the performance of the network such as delay and packet delivery ratio than traditional routing algorithms. [6]

Ant colony optimization (ACO) takes inspiration from the foraging behaviour of real ant species. This ACO exploits a similar mechanism for solving optimization problems for the various engineering field [8]. Finally it presents the experimental result of ACO which is applied for routing problem and compared with existing algorithms.

A new proposal of using particle swarm optimization algorithms to solve multi-objective optimization problems is presented. The algorithm is constructed based on the concept of Pareto dominance, as well as a state-of-the-art 'parallel' computing technique that intends to improve algorithmic effectiveness and efficiency simultaneously. The proposed parallel particle swarm multi-objective evolutionary algorithm (PPS-MOEA) [9] is tested through a variety of standard test functions its performance is compared with six noted multiobjective algorithms. The computational experience gained from the first two experiments indicates that the algorithm proposed in this article is extremely competitive when compared with other MOEAs, being able to accurately, reliably and robustly.

Inspired by swarm intelligence observed in social species, the artificial self-organized networking (SON) [10] systems are expected to exhibit some intelligent features (e.g., flexibility, robustness, decentralized control, and self-evolution, etc.) It is concluded that the bio-inspired mechanism is indeed a powerful source of innovative networking paradigm for artificial SON systems.
Bee swarms [13] exhibit much intelligent behaviour in their tasks such as nest site building, marriage, foraging, navigation and task selection. There is an efficient task selection mechanism in a bee swarm that can be adaptively changed by the state of the hive and the environment. Foraging is another crucial task for bees. Forage selection depends on recruitment for and abandonment of food sources. Communication among bees related to the quality of food sources takes place in the dancing area. Various dances are performed on the dancing area, such as waggle, round, tremble depending on the distance of the discovered source. [11]

Solving the fundamental transportation problems by designing intelligent local rules for vehicles approaching intersections by taking inspirations from self organizing biological systems(ants, bees, termites, fish, birds etc).[15]

To investigate the usefulness of the different approaches adopted by the algorithms when confronted with the peculiarities of urban environments and the requirements of real-world applications. Model node mobility by limiting node movements to the street and open spaces of town, use a ray-tracing approach to model the propagation of radio waves, and investigate different kinds of interactive data traffic patterns, ranging from SMS messaging to VoIP communications. Swarm intelligence based routing algorithm performs better than proactive and reactive routing algorithm. [16]

Swarm intelligence [7] a new approach for an on demand adhoc routing algorithm is proposed. The key components of swarm intelligence are self-organization and division of labour. In a self-organizing system, each of the covered units may respond to local stimuli individually and act together to accomplish a global task via division of labour without a centralized supervision. The entire system can adapt to internal and external changes efficiently. Four basic properties on which self-organization rely: positive feedback, negative feedback, fluctuations and multiple interactions [17]. Positive feedback means that an individual recruits other individuals by some directive, such as dancing of bees in order to lead some other bees onto a specific food source site. Negative feedback avoids all individuals accumulating on the same task by rebalancing the attraction negatively, such as abandoning the exhausted food source. Fluctuations are random behaviours of individuals in order to explore new states, such as random flights of scouts in a bee swarm. Multiple interactions are the basis of the tasks to be carried out by certain rules [18]

Wireless sensor networks (WSNs) [19] consist of a large number of autonomous nodes equipped with sensing capabilities, wireless communication interfaces, limited processing and energy resources. In WSN, the restrictions on energy efficiency are more compelling, nodes are usually static, and the networks are assumed to be much larger. Manet's traffic patterns strictly depend on the application and are address centric; in WSNs they are usually data-centric and are in the form of direct and reversed multicast trees rooted at monitor nodes. SI-based WSNs routing lacks of contributions falling in the two opposite areas of mathematical modelling and real-world implementations.

Swarm intelligence (SI) which is an artificial intelligence discipline and concerned with the design of intelligent multiagent systems by taking inspiration from the collective behaviour of social insects such as ants, termites, bees, and wasps, as well as from other animal societies such as flocks of birds or schools of fish. [18] 
The ability of Particle Swarm Optimization (PSO), heuristic technique for search of optimal solutions based on the concept of swarm. PSO reveals itself very effective in facing multivariable problems in which any variable takes on real values. A plan to endow PSO with fitness sharing which helps in improving performance of routing protocols.

Nature's self-organizing systems like insect societies show they can self organize and self configure. Making use of a number of relatively simple biological agents (e.g., the ants) a variety of different organized behaviours are generated at the system-level from the local interactions among the agents and with the environment. The robustness and effectiveness of such collective behaviours with respect to variations of environment conditions are key-aspects of their biological success. These kinds of systems are referred as Swarm Intelligence. Swarm systems have become a source of inspiration for the design of distributed and adaptive algorithms, and in particular of routing algorithms. Routing is the task of directing data flows from sources to destinations maximizing network performance.

Self-organization [20] existing in many branches of science such as biology, economic, telecommunications, etc., is essentially an interdisciplinary and heterogeneous research field. Self-organization is defined as "the emergence of system wide adaptive structure and functionality from simple local interactions between individual entities". Some appealing self-organization characteristics, such as "ability to self-organize in a fully distributed fashion, collaboratively achieving efficient equilibrium", have been generalized from biological systems and processes self-organization being observed in nature and finding their roots in biological mechanisms can be named bio-inspired. Inspired by swarm intelligence observed in social species, the artificial selforganized networking (SON) [10] systems are expected to exhibit some intelligent features (e.g., flexibility, robustness, decentralized control, and self-evolution, etc.). The bioinspired mechanism is indeed a powerful source of innovative networking paradigm for artificial SON systems.

\section{CONCLUSION}

MANET consists of a set of mobile nodes and challenges in routing due to dynamic network topology, power conservation in wireless nodes. The characteristics of MANET such as bandwidth constrained, energy constrained, limited physical security and dynamic network topology etc. Randomized change of domain causes change in topology of networks. Hence the major issue related to MANETs is routing. The proactive and reactive routing algorithms have their own limitations and are not found suitable for routing in MANET mainly when real time applications. The aim of the study is to investigate the advantages of the different approaches in relation to the characteristics of urban environments and to concrete application models for real-world MANETS.

SI based algorithms such as PSO, ACO ,SON and honey bees, nature inspired routing algorithms are critical to analyse and their appropriateness is observed. Si based algorithms are useful for specific nature of Ad-hoc networks due to free mobility and frequent topology changes. The multi-objective constraints based issues like mobility, path optimization, resource utilization and energy awareness are effectively solved by exploiting SI based metaheuristics in MANET's.

Swarm intelligence based routing algorithm are suitable for specific nature of ad- hoc networks in which environmental factors effects real time applications. The survey provides collection of swarms intelligence based algorithms for mobile ad-hoc network and their critical analysis. Real scenarios/constraints/environmental conditions are need to tune and simulate to get an efficient and effective routing protocol for MANET.

\section{REFERENCES}

[1] Siva. R.M, C. and B. S. Manoj, "Ad-Hoc Wireless Networks: Architecture And Protocols", Pearson, USA, 2004.

[2] E. Royer and C. K. Toh, "A review of current routing protocols for ad-hoc mobile wireless networks",IEEE Personal Communications, 1999.

[3] Rajagopalan. R, Dahlstrom,"Performance comparison of routing protocols in mobile ad hoc networks in the presence of faulty nodes", \{IEEE International Conference On Wireless Information Technology And Systems (ICWITS) \}, 11-16 NOV. 2012.

[4] Xiaodong Li, Xin Yao, "Cooperatively Coevolving Particle Swarms for Large Scale Optimization", \{IEEE Transactions On Evolutionary Computation\}, VOL. 16, NO. 2, APRIL 2012.

[5] Rubio largoA, Vega RodriguezM, Gomez Pulido, Sanchez-Perez, "Comparative Study on Multi objective Swarm Intelligence for the Routing and Wavelength Assignment problem", \{ IEEE Transactions On Systems, Man And Cybernetics Part C\}, Volume:42, Issue: 6 , Nov 2012.

[6] Asadinia. S, Rafsanjani. M.k , Saeid. A.B, "A novel routing algorithm based-on ant colony in Mobile Ad hoc Networks", \{ 3rd IEEE International Conference on Ubi-media Computing (U-Media)\}, 5-6 July 2010.

[7] G. Di Caro, F. Ducatelle, L. M. Gambardella, "Swarm Intelligence for routing in mobile ad hoc networks", $\{$ In Proceedings of the 2005 IEEE Swarm Intelligence Symposium, SIS 2005\}, pp. 76-83, 2005.

[8] B. Chandra Mohan \& R. Baskaran, "Survey on Recent Research and Implementation of Ant Colony Optimization in Various Engineering Applications", \{International Journal of Computational Intelligence Systems Taylor And Franchis\}, Volume 4, Issue 4, 2011.

[9] Shu-Kai S. Fana \& Ju-Ming Changa, "A parallel particle swarm optimization algorithm for multi-objective optimization problems", \{Engineering Optimization Taylor and Franchis\},Volume 41, Issue 7, 2009.

[10] Zhang, Z. ; Long, K. ; Wang, J. ; Dressler, F ,"On Swarm Intelligence Inspired Self-Organized Networking: Its Bionic Mechanisms, Designing Principles and Optimization Approaches", Communications Surveys \& Tutorials, IEEE, ( Issue: 99 ), 04 July 2013.

[11] Bahriye Akay, Dervis Karaboga, "A modified Artificial Bee Colony algorithm for real-parameter optimization", \{ Elsevier\}, 2010.

[12] F. Ducatelle, G. D. Caro, and L. M. Gambardella, "Using ant agents to combine reactive and proactive strategies for routing in mobile ad hoc networks", \{International Journal Of Computational Intelligence And Applications \}, vol. 5, no. 2, pp. 169-184, 2005.

[13] F. Dressler and O. Akan,"Bio-inspired networking: from theory to practice",\{IEEE Communication Magzine.\}, vol. 48, no. 11, pp. 176-183, 2010 
[14] F. Dressler and O. Akan, "A Survey on Bio-inspired Networking", \{Elsevier Computer Networks\}, vol. 54, no. 6, pp. 881-900, 2010.

[15] O. Tonguz, "Biologically inspired solutions to fundamental transportation problems", \{IEEE Communication. Magazine\}, vol. 49, no. 11, pp. 106115, Nov. 2011.

[16] Frederick Ducatelle, Gianni A. Di Caro, Luca M. Gambardella," An Evaluation of Two Swarm Intelligence MANET Routing Algorithms in an Urban Environment", \{IEEE Swarm Intelligence Symposium\}, 2010.

[17] Roth, M., "Termite: A Swarm Intelligent Routing Algorithm for Mobile Wireless Ad-Hoc Networks", \{Thesis and Dissertations\}, Cornell University Graduate School (2005).
[18] Ajith Abraham, Crina Grosan, Vitorino Ramos, "Stigmergic Computational Intelligence", \{Spinger\},2010.

[19] Muhammad Saleem a, Gianni A. Di Caro b, Muddassar Farooq, "Swarm intelligence based routing protocol for wireless sensor networks: Survey and future directions", \{Information Sciences Elsevier\}, 2010.

[20] Falko Dresser, "A Study of Self Organization Mechanism in Ad-hoc and Sensor Networks", \{Elsevier Computer Communications \}, Vol. 31(13), 2008.

[21] S. Lalwani, S. Singhal, R. Kumar and N. Gupta, "A Comprehensive Survey: Applications Of Multi-Objective Particle Swarm Optimization (Mopso) Algorithm", \{Transactions On Combinatorics\}, Vol. 2 No. 1 (2013), pp. 39-101. 